\title{
Spatial Factors Affecting the Frequency of Pedestrian Traffic Crashes: A Systematic Review
}

\author{
Ali Moradi, ${ }^{1}$ Hamid Soori, ${ }^{2,}$ Amir Kavousi, ${ }^{3}$ Farshid Eshghabadi, ${ }^{4}$ and Ensiyeh Jamshidi ${ }^{5}$ \\ ${ }^{1}$ Department of Epidemiology, Faculty of Public Health, Shahid Beheshti University of Medical Sciences, Tehran, IR Iran \\ ${ }^{2}$ Safety Promotion and Injury Prevention Research Center, Shahid Beheshti University of Medical Sciences, Tehran, IR Iran \\ ${ }^{3}$ School of Health, Safety and Environment, Shahid Beheshti University of Medical Sciences, Tehran, IR Iran \\ ${ }^{4}$ Department of Human Geography/Urban Planning, Faculty of Geography, University of Tehran, Tehran, IR Iran \\ ${ }^{5}$ Community Based Participatory Research Center, Iranian Institute for Reduction of High-Risk Behaviors, Tehran University of Medical Sciences, Tehran, IR Iran \\ "Corresponding author: Hamid Soori, Safety Promotion and Injury Prevention Research Center, Shahid Beheshti University of Medical Sciences, Tehran, IR Iran. Tel: \\ +98-2122439980, E-mail: hsoori@yahoo.com
}

Received 2015 June 15; Revised 2016 May 30; Accepted 2016 June 19.

\begin{abstract}
Context: Considering the importance of pedestrian traffic crashes and the role of environmental factors in the frequency of crashes, this paper aimed to review the published evidence and synthesize the results of related studies for the associations between environmental factors and distribution of pedestrian-vehicular traffic crashes.

Evidence Acquisition: We searched all epidemiological studies from 1966 to 2015 in electronic databases. We found 2,828 studies. Only 15 observational studies out of these studies met the inclusion criteria of the study. The quality of the included studies was assessed using the strengthening the reporting of observational studies in epidemiology (STROBE) checklist.

Results: A review of the studies showed significant correlations between a large number of spatial variables including student population and the number of schools, population density, traffic volume, roadway density, socio-economic status, number of intersections, and the pedestrian volume and the dependent variable of the frequency of pedestrian traffic crashes. In the studies, some spatial factors that play an important role in determining the frequency of pedestrian traffic crashes, such as facilities for increasing the pedestrians' safety were ignored.

Conclusions: It is proposed that the needed research be conducted at national and regional levels in coordination and cooperation with international organizations active in the field of traffic crashes in various parts of the world, especially in Asian, African and Latin American developing countries, where a greater proportion of pedestrian traffic crashes occur.
\end{abstract}

Keywords: Pedestrian, Road Crashes, Spatial Analysis, Spatial Factors, Systematic Review

\section{Context}

Traffic crashes are considered to be the leading cause of death and disability in the world (1). They result in 1.2 million annual deaths and cause injury to tens of millions of people around the world (2). A significant proportion of deaths and injuries results from traffic crashes among pedestrians. Around the world, an annual 400,000 pedestrians lose their lives due to traffic crashes (3). In recent years, pedestrian deaths and injuries caused by traffic crashes have increased globally (4).

The ratio of pedestrian fatalities to total fatalities related to traffic crashes is different in each country. This ratio is much higher in developing countries than that in developed countries. In Peru, Mozambique, and El Salvador, $86 \%, 68 \%$, and $63 \%$ of deaths resulting from traffic crashes occur to pedestrians, respectively. In the United States this ratio is equal to $13 \%$ (5), and pedestrian traffic injuries ac- count for 1.65 deaths and 22 cases of hospitalization out of every 100,000 people in the country (6). In $2013,22 \%$ of all fatalities as a result of road traffic crashes in the EU were fatalities of pedestrians (7).

A significant proportion of pedestrian victims of traffic crashes are children and teenagers. Children account for approximately $21 \%$ of fatalities from pedestrian traffic crashes globally (8).

Models for estimating the frequency of traffic crashes in a specific geographical area and time are widely used. One of the main uses of these models is to rate the different regions in terms of traffic crashes, which aims to identify areas with high risk and lack of safety facilities. Ranking the regions in terms of safety is one of the crucial factors in the engineering design of transport networks (9). 


\subsection{Objectives}

Given the importance of these models and due to the lack of review on studies using statistical models to determine the role of environmental factors in the frequency of traffic crashes in geographical units, this study aimd to summarize and analyze the results of these studies by performing a systematic review (10).

\section{Evidence Acquisition}

We sought to identify all epidemiological studies, which determined the correlation between different spatial and environmental attributes and pedestrianvehicular crashes in geographic units. The question addressed in this systematic review was: which spatial and environmental attributes are associated with pedestrianvehicular crash counts?

Studies were included in the review if they were observational and assessed the correlation of one or more spatial and environmental attributes and pedestrianvehicular crash counts in geographical units. We excluded interventional studies, case reports, case series, and studies that assessed correlation of pedestrian-vehicular crash counts and merely demographic attributes such as sex and age.

Search strategy and selection criteria: We followed a standard protocol for doing a systematic review: a computerized search was performed using databases including Medline (1966 - 2015), Sciencedirect (1970 - 2015), Scopus (1970 - 2015), Embase (1966 - 2015), the Cochrane Library (1966 - 2015) and ISI Web of Knowledge (1966 - 2015). Web sites of institutions involved in research and policy in the areas of road safety such as road traffic injuries research network (RTIRN) were searched up until March 2015. Reference lists of identified articles and proceedings of relevant conferences were hand-searched. The review was not restricted to published or peer-reviewed literature. Electronic databases were searched using the following keywords: pedestrian and crashes, pedestrian and vehicle collisions, pedestrian and injury, pedestrian and casualties, pedestrian and hazard, pedestrian and risk factor, pedestrian and safety, pedestrian and security, pedestrian and spatial analysis, pedestrian and spatial attributes, pedestrian and geographical attributes, pedestrian geographical units, pedestrian and environmental characteristics, pedestrian and built environment, pedestrian and land use, pedestrian and road segments, pedestrian and traffic, pedestrian and transportation, and pedestrian and road segments.

Quality Assessment of the Articles: Articles published in indexed and peer-reviewed journals were considered to be high-quality articles. The quality of articles published in non-indexed journals, reports and books published by UN agencies (WHO, UNESCO, UNICEF) and the World Bank, as well as in the proceedings of conferences and seminars were assessed by experts in the epidemiology of traffic crashes and by using the strengthening the reporting of observational studies in epidemiology (STROBE) checklist.

Data Analysis: Titles and abstracts of the articles were reviewed by two separate individuals. Articles and reports that were deemed irrelevant by these two reviewers were excluded from the study list. The number of excluded articles and their titles were recorded. Then, the full text of articles and reports on the agenda were retrieved and referred to two independent teams of reviewers. Each team separately extracted the answers to the research question from the contents of the paper and took necessary notes. If there were any differences in the results recorded by the two teams, the original text of the article was evaluated by the project supervisor who then made a decision on the data of the articles at hand.

\section{Results}

In the search we found 2,828 studies. Of these studies, 2,784 titles were unrelated to the objectives of the study and were excluded. After a critical appraisal of the remaining 44 articles, there were 15 studies that met the inclusion criteria. A flowchart of the systematic review process for determining the correlation of different spatial and environmental attributes and pedestrian-vehicular crashes in populated areas is illustrated in Figure 1. All retrieved studies were conducted between 2000 and 2014 and used crosssectional design. Characteristics of the studies included in the systematic review are summarized in appendix 1 . The definitions of some of the geographical units is presented in Table 1. Also, the definitions of some independent variables are presented in Table 2 .

Correlations of spatial variables such as population of students or the number of schools, population density, traffic volume, roadway density, socio-economic status of the region, number of intersections and pedestrian volume with the dependent variable (crash count) have been investigated in an increasing number of studies that are briefly mentioned below.

\subsection{Student Population or the Number of Schools}

In 6 out of the 15 studies, the frequency of pedestrian traffic crashes and the number of schools were investigated. Several indicators, such as log of enrolment, proportion of children per catchment area, total number of schools, child population as a proportion of total population (\%), and log of child population ages 5 - 14 were used 
Table 1. Definition of Some of the Geographical Units Used in the Reviewed Articles

\begin{tabular}{|c|c|}
\hline Geographical Unit & Definition \\
\hline Census tracts & $\begin{array}{l}\text { Census tracts are small, relatively permanent statistical subdivisions of a county or equivalent entity that are updated by local } \\
\text { participants prior to each decennial census as a part of the Census Bureau's Participant Statistical Areas Program. Census tracts } \\
\text { generally have a population size between } 1,200 \text { and } 8,000 \text { people, with an optimum size of } 4,000 \text { people. A census tract usually } \\
\text { covers a contiguous area; however, the spatial size of census tracts varies widely depending on the density of settlement. Census } \\
\text { tract boundaries are delineated with the intention of being maintained over a long time so that statistical comparisons can be } \\
\text { made from census to census. }\end{array}$ \\
\hline Census block group & $\begin{array}{l}\text { A census block group is a fairly small geographic area (the average area of census block groups is } 4.125 \text { square kilometers in the } \\
\text { Hillsborough County). It is used as a spatial analysis unit. }\end{array}$ \\
\hline School buffer zone & Area within a quarter mile buffer of a public school \\
\hline School zone & Area near a school \\
\hline Lower super output area (LSOA) & A geography developed by grouping together a small number of Output Areas (the base unit for the release of census data). \\
\hline Traffic analysis zone(TAZ) & $\begin{array}{l}\text { A TAZ is a spatial aggregation of census blocks and its size is in part a function of population. TAZs are special areas delineated by } \\
\text { state and/or local transportation officials particularly for tabulating traffic-related data. They are also defined as part of the } \\
\text { Census Transportation Planning. TAZs include spatial contiguity, homogeneity, and compactness. Homogeneity of a TAZ is } \\
\text { defined by a single predominant land use and homogeneous socio-economic characteristics. }\end{array}$ \\
\hline Near school area & Area within a one-mile buffer of a school \\
\hline
\end{tabular}

Table 2. Definition of Some of the Independent Variables Used in the Reviewed Articles

\begin{tabular}{|c|c|}
\hline Independent Variable & Definition \\
\hline Social deprivation index & $\begin{array}{l}\text { This index includes five variables: the percentage of single-parent families, the unemployment rate, the percentage of adults } \\
\text { age } 20 \text { and over with less than a ninth grade education, the percentage of low income population, and the percentage of } \\
\text { immigrants having arrived during the period 1996-2001. The proposed deprivation index represents the sum of these five } \\
\text { standardized variables ( } 0 \text { to } 1 \text { scale) and it varies from minimal deprivation (0) to maximal deprivation (5). }\end{array}$ \\
\hline Walking to work & Proportion of labor force aged $\geq 16$ y who walk or take public transport as their main mode of transportation to work in tract \\
\hline Density of liquor license outlets & Total liquor license outlets per 1000000 square feet in tract \\
\hline Transit availability index (TAI) & $\begin{array}{l}\text { Composite index giving the extent to which residents have access to transit (bus and rail); based on three input measures of } \\
\text { frequency (person-minutes served), hours of service (number of hours), and service coverage (percentage of census tract area } \\
\text { covered). }\end{array}$ \\
\hline Pedestrian accessibility index (PED) & $\begin{array}{l}\text { Composite index ranking tract suitability for pedestrian travel; based on input values of population, income, number of } \\
\text { households, amount of commercial and residential land uses as a percentage of census tracts, weighted trip origins and } \\
\text { destinations, and pedestrian environment factor (PEF) values, where PEF's are the average number of blocks for the quarter } \\
\text { section within each census tract and the eight adjacent quarter sections. }\end{array}$ \\
\hline Sum of annual average daily traffic & $\begin{array}{l}\text { Total annual average daily traffic on links of all highway functional classes within census tract; output from a regional traffic } \\
\text { assignment model and geographical information system (GIS) }\end{array}$ \\
\hline Crime rate & Total violent and nonviolent crimes in census tracts \\
\hline Intersection indicator & The type of intersections (e.g., three-legged versus four legged intersection) \\
\hline Length of basic spatial units & $\begin{array}{l}\text { The socioeconomic deprivation index was derived from the } 2006 \text { by-census. The Census and Statistics Department of Hong } \\
\text { Kong conducts a population census every ten years and a by-census in the middle of the intercensal period. In this study, } \\
\text { some indicators were extracted to describe socioeconomic conditions of each TPU from the } 2006 \text { by-census reports. The } \\
\text { variables are based on previous literature on area deprivation predictors of pedestrian casualties. The indicators on income, } \\
\text { owner-occupancy, education, occupation and unemployment are defined as follows: (a) Income: monthly household income } \\
\text { <6000 HKD (\%);(b) Owner-occupancy: not owner-occupied households (\%);(c) Education: low upper-secondary education } \\
\text { attainment (\%);(d) Occupation: occupation with no or low qualifications (\%); and(e) Unemployment: unemployment (\%). } \\
\text { Depending on these five predictors, the socioeconomic deprivation index (SDI) was calculated using the Z-scores method }\end{array}$ \\
\hline
\end{tabular}

in these studies. In 5 studies, the correlation between frequency of pedestrian traffic crashes and the mentioned independent variables was statistically significant (11-15) and the significance level ranged from $\mathrm{P}<0.05$ to $\mathrm{P}<0.0001$. In a study conducted by Green et al. (12), the significance level was reported to be higher than that of other studies $(\mathrm{P}<$ 0.0001). The statistical population in this study consisted of children less than 17 years of age, and the child population in proportion to the total population (\%) was considered to be the independent variable. The direction of correlation was positive in 4 of the retrieved studies $(11,12,14$,
15). In other words, most of these studies showed that frequency of pedestrian traffic increases when there is an increase in the student population or the number of schools.

\subsection{Population Density}

In 6 of the 15 studies, the frequency of pedestrian traffic crashes and the population density were assessed. Several independent variables, such as population, population density and log of population density were used. In 5 studies, the correlation of frequency of pedestrian traffic crashes and the mentioned independent variables was 


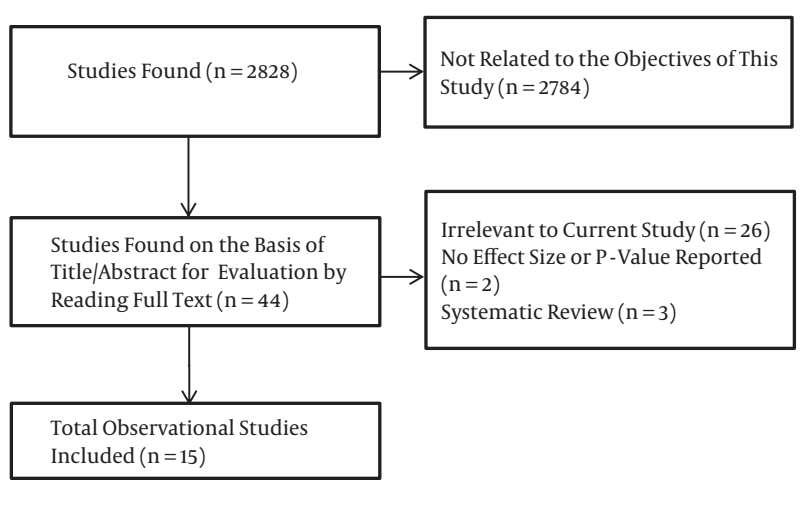

Figure 1. Flow Diagram of Systematic Review to Identify Eligible Studies

statistically significant (14-18). In a study conducted by Siddiqui et al. (17), the significance level was reported to be higher than that in other studies $(\mathrm{P}<0.0001)$. The statistical population was the total population, and log of population density was used as the independent variable. The direction of correlation was similar and positive in all studies. In other words, all 5 studies showed that with the increase in population density, the frequency of pedestrian traffic crashes also increases.

Traffic Volume: In 6 of the 15 reviewed studies, the relationship between the frequency of pedestrian-related traffic crashes and traffic volume was investigated. These studies used different indicators for traffic volumes including traffic volume, sum of annual average daily traffic, Ln (average annual daily traffic), average daily traffic flow $\times 1000$ and Ln (daily vehicle volume) $(11,13,19-22)$. In all 6 studies, the correlation of the mentioned variables was statistically significant and the significance level ranged from $\mathrm{P}<0.04$ to $\mathrm{P}<0.0001$. In studies conducted by Wier et al., and Schneider et al. $(20,22)$, the significance level were reported to be higher than that in other studies (P $<0.0001)$. The statistical population in these studies was the total population and pedestrians of University of North Carolina at Chapel Hill (UNC), respectively. The direction of correlation in all studies was similar and positive. In other words, all 6 studies showed that with the increased traffic volume, the frequency of pedestrian traffic crashes also increases.

\subsection{Roadway Density}

In 6 of the 15 studies, the frequency of pedestrian traffic crashes and roadway density were investigated. These studies used independent variables such as roadway density and roadway length. In 5 studies, the relationship of the mentioned variables was statistically significant and the significance level ranged from $\mathrm{P}<0.05$ to $\mathrm{P}<0.0001$ (11,
$12,17,21,23)$. In a study conducted by Garder et al. (23), the significance level was reported to be higher than that in other studies $(\mathrm{P}<0.0001)$. In this study, the statistical population was the total population, and the roadway length was used as an independent variable. The direction of correlation in the two studies was statistically significant and positive $(12,17)$. In the study conducted by Cottrill et al. (11), the direction of correlation was negative and in the study conducted by Garder et al., the direction of correlation was not reported (23).

Socioeconomic Status of the Region: In 5 of the 15 studies, the frequency of pedestrian traffic crashes and socioeconomic status was investigated. These studies used different indicators for socioeconomic status including social deprivation index, median household income and median income $\times 10000$. In 4 studies, the relationship of the mentioned variables was statistically significant and the significance level ranged from $\mathrm{P}<0.05$ to $\mathrm{P}<0.0001(15,17,24$, $25)$. In the studies conducted by Cloutier et al., and Siddiqui et al. $(17,25)$, a greater significance level was reported compared to that of other studies $(\mathrm{P}<0.0001)$. The statistical population in the former study was composed of child pedestrians aged 5 to 14 years, and in the latter study, the total population was the statistical population. The direction of correlation in all of the studies was negative. In other words, all studies showed that with the improving socioeconomic status, the frequency of pedestrian traffic crashes was reduced.

\subsection{The Number of Intersections}

In 5 of the 15 studies, the frequency of pedestrian traffic crashes and the number of intersections were assessed. These studies used different indicators including intersection indicator, total number of intersections, Ln (segment/intersection length) and cross-streets per kilometer roadway. In 4 studies, the relationship of the mentioned variables was statistically significant and the significance level ranged from $\mathrm{P}<0.05$ to $\mathrm{P}<0.0001(13,17,20,24)$. In the studies conducted by Schneider et al. and Siddiqui et al. $(17,25)$, a greater significance level was reported compared to that of other studies $(\mathrm{P}<0.0001)$. The statistical population in the former study composed of pedestrians of UNC campus, and in the latter study, the total population was the statistical population. The Ln (segment/intersection length) and the total number of intersection were used as independent variables, respectively. The direction of correlation in all studies was similar and positive. In other words, all 4 studies showed that by increasing the number of intersections, the frequency of pedestrian traffic crashes was also increased. 


\subsection{Commercial Land Use}

In 5 of the 15 studies, the frequency of pedestrian traffic crashes and commercial land use were investigated. These studies used different indicators including commercial land use/total land use, commercial buffer, neighborhood commercial area and commercial access. In 4 studies, the relationship of the mentioned independent variables was statistically significant and the significance level ranged from $\mathrm{P}<0.05$ to $\mathrm{P}<0.0001(13,14,21,22)$. In a study conducted by Wier et al. (22), a greater significance level was reported compared to that of other studies $(\mathrm{P}<$ $0.0001)$. The direction of correlation in all studies was similar and positive. In other words, all 4 studies showed that with the increasing commercial land use, the frequency of pedestrian traffic crashes was also increased.

\subsection{Pedestrian Volume}

In 3 of the 15 studies, the frequency of pedestrian traffic crashes and pedestrian volume were assessed. In all 3 of these studies, the correlation of frequency of pedestrian traffic crashes and the mentioned independent variables was statistically significant and the significance level ranged from $\mathrm{P}<0.01$ to $\mathrm{P}<0.0001(13,19,20)$. In the studies conducted by Schneider et al. (20), a greater significance level was reported compared to that of other studies $(\mathrm{P}<0.0001)$. The statistical population of this study was the pedestrians of UNC campus. The direction of correlation was similar and positive in all studies. In other words, all 3 studies showed that with increase in pedestrian volume, the frequency of pedestrian traffic crashes was also increased.

Furthermore, the correlations of other independent variables such as speed, roadway width, signal, park land, main road density (log), land area, walking to work, liquor license outlet density, transit availability index (TAI), crime rate, area of domestic gardens, average weighted distance to key services, pedestrian traffic flow, dwelling unit, number of crosswalks per 1000 linear feet, and pedestrian accessibility index (PED) and frequency of pedestrian-related traffic crashes were reviewed in retrieved articles. In most cases, these correlations were statistically significant.

\section{Conclusions}

This systematic review of studies was conducted to assess the role of spatial and environmental factors in the frequency of pedestrian-vehicular traffic crashes in geographical units. In the studies included in the systematic review, a relatively wide range of spatial risk factors affecting pedestrian traffic crashes was investigated. Most of the studies included in this systematic review were conducted in the United States and geographic units compared in most studies were census tracts. In most studies, the negative binomial regression models or generalized negative binomial models were used to measure the correlation between dependent and independent variables. Also, in most studies the statistical population consisted of the total population of the area, and in a number of studies, the statistical population consisted of school-age children. Our systematic review showed that in most statistical models, the increase in student population, the number of schools, commercial land use, the number of intersections, and roadway density are among the main factors behind the increased number of pedestrian traffic crashes.

It is noteworthy that 11 of 15 retrieved studies were conducted in the United States, 2 of the remaining 4 studies were conducted in Canada, 1 study was conducted in Europe, and 1 study in Asia in the city of Hong Kong, which is considered a developed city. Despite great care in reviewing the literature, no study was found to be conducted in developing countries. Therefore, this systematic review suggests that although various studies showed that most of the injuries and deaths of pedestrians caused by traffic crashes occurred in developing countries $(3,5,10,26,27)$, it is necessary to conduct research on risk factors for these types of crashes in these countries.

On the other hand, given that most of the studies included in this review were conducted in metropolises of developed countries, the results cannot be generalized to all urban areas; especially cities with different structures in Asia and Latin America, and consequently, it can cause differences in the number of spatial risk factors for pedestrian traffic crashes.

Furthermore, most of these studies were conducted in the metropolitan areas of the United States and Canada and in all of these studies; the area studied was a city or part of a city. Even in the studies conducted in these developed countries, only inter-urban traffic crashes in the metropolises were considered and few studies were conducted on the frequency of these type of crashes in the suburban streets and smaller population centers.

This systematic review showed that in all studies in which the variables of population density, traffic volume, and the volume of pedestrians were included in the final models, there was a significant relationship with the dependent variable of pedestrian traffic crash counts. The direction of correlation of variables was also positive in all models. In other words, review of various studies showed that by increasing the population density, the traffic volume, as well as the pedestrian volume, the frequency of pedestrian traffic crashes was also increased. Therefore, these variables can be considered as key factors in deter- 
mining the frequency of pedestrian traffic crashes. It is necessary to conduct studies on the mechanism of their effects on pedestrian-vehicular traffic crashes.

The number of pedestrian traffic crashes is higher in 4 of 5 studies conducted on the relationship between socioeconomic status and the number of pedestrian traffic crashes $(15,17,24,25)$. In recent years, most studies that investigated the inequity in traffic crashes also showed that in poorer areas, the number and severity of traffic crashes are higher (28-34). Further studies should be conducted in this field, especially in metropolises of developing countries.

Several studies revealed that driving over the speed limit is one of the most important risk factors associated with the increased severity of pedestrian traffic crashes (35-42). Therefore, although this risk factor may increase the frequency of these crashes, only one of the studies included in this systematic review investigated the relationship between speeding and pedestrian traffic crashes counts. This correlation was also highly statistically significant. Therefore, it can be said that in the studies conducted in this field, the effect of this important risk factor on the number of pedestrian traffic crashes was not studied and is considered to be one of the major weaknesses of these studies.

Furthermore, in the studies included in the systematic review, not enough attention has been paid to the role of urban and suburban roadway safety facilities, such as pedestrian bridges, lining of pedestrians crossing, warning signs, etc., in the frequency of pedestrian traffic crashes. The only studies to look at these types of variables were conducted by Schneider et al., where the correlation of pedestrians' crossing points was assessed, and in the study conducted by Garder et al, where the correlation of traffic signs with the frequency of pedestrian traffic crashes was investigated $(20,23)$. Considering that these types of safety facilities are of spatial factors that can be associated with the frequency of pedestrian traffic crashes, it is necessary to consider this issue in future studies.

All studies that met the inclusion criteria of the systematic review were conducted as cross-sectional. What is missing is the study of causal reasoning and the provision of higher levels of evidence by using cohort and casecontrol methodology to identify risk factors for pedestrian traffic crashes. Therefore, organizations and institutions that are active in the research and prevention of traffic crashes should consider this issue and examine the risk factors for these crashes by conducting studies that provides stronger causal reasoning.

In this systematic review, in most cases (11 of the 15 studies), the statistical population was the total population; and only 4 of the studies conducted to identify risk factors of traffic crashes related to the school-age child pedestrians met the criteria for inclusion in the systematic review. However, according to the World Health Organization, approximately $21 \%$ of deaths from pedestrian traffic crashes occur to children (6). In addition, various studies showed that children, especially school-age children, are one of the groups at the most risk for such crashes (43-45). Therefore, relatively few studies conducted on the spatial risk factors for the pedestrian traffic crashes were focused on children. Hence, it is necessary to consider this issue in future studies on the spatial risk factors associated with the frequency of pedestrian traffic crashes.

Among the 15 studies reviewed, the studies conducted by Schneider and colleagues and by Miranda-Moreno et al., the role of the number of bus stops was assessed $(13,20)$, and only in the study conducted by Clifton et al. (18), the role of the parking areas in the frequency of pedestrian traffic crashes was evaluated. No study addressed the taxi stations. This shows that in the studies that were done in this regard little attention was paid to the role of these variables in the frequency of pedestrian traffic crashes. Because there is usually a high volume of pedestrians in the streets around bus stops, taxi stations, and public parking lots, the study of the distribution of the pedestrian traffic crashes using methods like spatial analysis and regression models can clarify the role of these spatial factors in the frequency of pedestrian traffic crashes.

All of the research conducted in recent years in the field of the correlation of spatial factors with the frequency of pedestrian traffic crashes was systematically reviewed. The results showed that few studies were conducted on this concept and the majority of the studies were carried out in developed countries, especially in the metropolises of the United States. In these studies, some spatial factors that play an important role in determining the frequency of pedestrian traffic crashes, such as facilities for increasing the pedestrians' safety were ignored. Strong causal inference schemes and advanced techniques of spatial analysis which use relevant software were not used and sufficient attention was also not paid to high-risk groups. Therefore, it is proposed that the needed research be conducted at national and regional levels in coordination and cooperation with international organizations active in the field of traffic crashes, such as the World Health Organization. This research should especially be conducted in Asian, African and Latin American developing countries because a greater proportion of pedestrian traffic crashes occurs in these countries. Prevention programs for reducing the frequency of pedestrian traffic crashes and their impact should be developed and implemented accordingly. 


\section{Supplementary Material}

Supplementary material(s) is available here.

\section{Acknowledgments}

The authors would like to thank the faculty members of the department of epidemiology and faculty of public health, Shahid Beheshti University of Medical Sciences, Tehran, Iran.

\section{Footnotes}

Authors' Contribution: Ali Moradi: design of the study and editing; Hamid Soori: supervisor and final editing; Amir Kavoosi: statistical advisor and final editing; Farshid Eshghbadi: geographic information system instructor; Ensiyeh Jamshidi: contributed to the editing of the article.

Funding/Support: This study was part of a thesis conducted in Shahid Beheshti University of Medical Sciences, Tehran, Iran, and was also funded by this university.

\section{References}

1. WHO . International statistical classification of diseases and related health problems(ICD 10). Geneva: World Health Organization; 2004.

2. WHO . Global status report on road safety. Geneva: World Health Organization; 2015.

3. Naci H, Chisholm D, Baker TD. Distribution of road traffic deaths by road user group: a global comparison. Inj Prev. 2009;15(1):55-9. doi: 10.1136/ip.2008.018721. [PubMed: 19190278].

4. Aidoo EN, Amoh-Gyimah R, Ackaah W. The effect of road and environmental characteristics on pedestrian hit-and-run accidents in Ghana. Accid Anal Prev. 2013;53:23-7. doi: 10.1016/j.aap.2012.12.021. [PubMed: 23357033].

5. Zegeer CV, Bushell M. Pedestrian crash trends and potential countermeasures from around the world. Accid Anal Prev. 2012;44(1):3-11. doi: 10.1016/j.aap.2010.12.007. [PubMed: 22062330].

6. Yanar H, Demetriades D, Hadjizacharia P, Nomoto S, Salim A, Inaba $\mathrm{K}$, et al. Pedestrians injured by automobiles: risk factors for cervical spine injuries. J Am Coll Surg. 2007;205(6):794-9. doi: 10.1016/j.jamcollsurg.2007.06.280. [PubMed: 18035263].

7. European Union . Road safety in the European Union: Trends, statistics and main challenges. Brussels, Belgium: European Commission; 2015.

8. Peden M. World report on child injury prevention. Geneva: World Health Organization; 2008.

9. Wang C, Quddus MA, Ison SG. Predicting accident frequency at their severity levels and its application in site ranking using a two-stage mixed multivariate model. Accid Anal Prev. 2011;43(6):1979-90. doi: 10.1016/j.aap.2011.05.016. [PubMed: 21819826].

10. WHO . Pedestrian safety: a road safety manual for decision-makers and practitioners. Geneva: World Health Organization; 2013.

11. Cottrill CD, Thakuriah PV. Evaluating pedestrian crashes in areas with high low-income or minority populations. Accid Anal Prev. 2010;42(6):1718-28. doi: 10.1016/j.aap.2010.04.012. [PubMed: 20728622].

12. Green J, Muir H, Maher M. Child pedestrian casualties and deprivation. Accid Anal Prev. 2011;43(3):714-23. doi: 10.1016/j.aap.2010.10.016. [PubMed: 21376859].
13. Miranda-Moreno LF, Morency P, El-Geneidy AM. The link between built environment, pedestrian activity and pedestrian-vehicle collision occurrence at signalized intersections. Accid Anal Prev. 2011;43(5):1624-34. doi:10.1016/j.aap.2011.02.005. [PubMed: 21658488].

14. Ukkusuri S, Miranda-Moreno LF, Ramadurai G, Isa-Tavarez J. The role of built environment on pedestrian crash frequency. Safety Sci. 2012;50(4):1141-51. doi: 10.1016/j.ssci.2011.09.012.

15. McArthur A, Savolainen PT, Gates TJ. Spatial Analysis of Child Pedestrian and Bicycle Crashes: Development of Safety Performance Function for Areas Adjacent to Schools.JTransport Res Board. 2014(2465):5763.

16. Sebert Kuhlmann AK, Brett J, Thomas D, Sain SR. Environmental characteristics associated with pedestrian-motor vehicle collisions in Denver, Colorado. Am J Public Health. 2009;99(9):1632-7. doi: 10.2105/AJPH.2007.131961. [PubMed: 19608966].

17. Siddiqui C, Abdel-Aty M, Choi K. Macroscopic spatial analysis of pedestrian and bicycle crashes. Accid Anal Prev. 2012;45:382-91. doi: 10.1016/j.aap.2011.08.003. [PubMed: 22269522].

18. Clifton KJ, Kreamer-Fults K. An examination of the environmental attributes associated with pedestrian-vehicular crashes near public schools. Accid Anal Prev. 2007;39(4):708-15. doi: 10.1016/j.aap.2006.11.003. [PubMed: 17174259].

19. Lascala EA, Gerber D, Gruenewald PJ. Demographic and environmental correlates of pedestrian injury collisions: a spatial analysis. Accid Anal Prev. 2000;32(5):651-8. [PubMed: 10908137].

20. Schneider RJ, Ryznar RM, Khattak AJ. An accident waiting to happen: a spatial approach to proactive pedestrian planning. Accid Anal Prev. 2004;36(2):193-211. [PubMed: 14642874].

21. Hashimoto T. Spatial analysis of pedestrian accidents [Dissertation]. US: University of South Florida; 2005.

22. Wier M, Weintraub J, Humphreys EH, Seto E, Bhatia R. An area-level model of vehicle-pedestrian injury collisions with implications for land use and transportation planning. Accid Anal Prev. 2009;41(1):13745. doi: 10.1016/j.aap.2008.10.001. [PubMed: 19114148].

23. Garder PE. The impact of speed and other variables on pedestrian safety in Maine. Accid Anal Prev. 2004;36(4):533-42. doi: 10.1016/S00014575(03)00059-9. [PubMed: 15094405].

24. Yao S, Loo BP. Identification of Hazardous Road Locations for Pedestrians.J Procedia Eng. 2012;45:815-23. doi: 10.1016/j.proeng.2012.08.244.

25. Cloutier MS, Apparicio P, Thouez JP. GIS-based spatial analysis of child pedestrian accidents near primary schools in Montréal, Canada. $J$ Appl GIS. 2007;3(4):1-18.

26. WHO . Global status report on road safety: Time for action. Geneva: World Health Organization; 2009.

27. WHO. Global status report on road safety: Supporting a decade of action. Geneva: World Health Organization; 2013.

28. Hasselberg M, Laflamme L, Weitoft GR. Socioeconomic differences in road traffic injuries during childhood and youth: a closer look at different kinds of road user. $J$ Epidemiol Community Health. 2001;55(12):858-62. [PubMed: 11707477].

29. Zambon F, Hasselberg M. Socioeconomic differences and motorcycle injuries: age at risk and injury severity among young drivers. A Swedish nationwide cohort study. Accid Anal Prev. 2006;38(6):1183-9. doi: 10.1016/j.aap.2006.05.005. [PubMed: 16806026].

30. Licaj I, Haddak M, Pochet P, Chiron M. Contextual deprivation, daily travel and road traffic injuries among the young in the Rhone Departement (France). Accid Anal Prev. 2011;43(5):1617-23. doi: 10.1016/j.aap.2011.02.003. [PubMed: 21658487].

31. Laflamme L, Engstrom K. Socioeconomic differences in Swedish children and adolescents injured in road traffic incidents: cross sectional study. BMJ. 2002;324(7334):396-7. [PubMed: 11850370].

32. Laflamme L, Diderichsen F. Social differences in traffic injury risks in childhood and youth-a literature review and a research agenda. Inj Prev. 2000;6(4):293-8. [PubMed: 11144632]. 
33. Morency P, Gauvin L, Plante C, Fournier M, Morency C. Neighborhood social inequalities in road traffic injuries: the influence of traffic volume and road design. Am J Public Health. 2012;102(6):1112-9. doi: 10.2105/AJPH.2011.300528. [PubMed: 22515869].

34. Laflamme L, Hasselberg M, Reimers AM, Cavalini LT, Ponce de Leon A. Social determinants of child and adolescent traffic-related and intentional injuries: a multilevel study in Stockholm County. Soc Sci Med. 2009;68(10):1826-34. doi: 10.1016/j.socscimed.2009.02.050. [PubMed: 19346046].

35. Kroyer HR. The relation between speed environment, age and injury outcome for bicyclists struck by a motorized vehicle - a comparison with pedestrians. Accid Anal Prev. 2015;76:57-63. doi: 10.1016/j.aap.2014.12.023. [PubMed: 25616032].

36. Liu Y-C, Tung Y-C. Risk analysis of pedestrians' road-crossing decisions: Effects of age, time gap, time of day, and vehicle speed. J Safety Sci. 2014;63:77-82. doi: 10.1016/j.ssci.2013.11.002.

37. Sasidharan L, Menendez M. Partial proportional odds model-an alternate choice for analyzing pedestrian crash injury severities. Accid Anal Prev. 2014;72:330-40. doi:10.1016/j.aap.2014.07.025. [PubMed: 25113015].

38. Kroyer HR. Is $30 \mathrm{~km} / \mathrm{ha}$ 'safe'speed? Injury severity of pedestrians struck by a vehicle and the relation to travel speed and age. IATSS Res.
2015;39(1):42-50.

39. Tefft BC. Impact speed and a pedestrian's risk of severe injury or death. Accid Anal Prev. 2013;50:871-8. doi: 10.1016/j.aap.2012.07.022. [PubMed: 22935347].

40. Aziz HM, Ukkusuri SV, Hasan S. Exploring the determinants of pedestrian-vehicle crash severity in New York City. Accid Anal Prev. 2013;50:1298-309. doi:10.1016/j.aap.2012.09.034. [PubMed: 23122781].

41. Kim JK, Ulfarsson GF, Shankar VN, Mannering FL. A note on modeling pedestrian-injury severity in motor-vehicle crashes with the mixed logit model. Accid Anal Prev. 2010;42(6):1751-8. doi: 10.1016/j.aap.2010.04.016. [PubMed: 20728626].

42. Rosen E, Sander U. Pedestrian fatality risk as a function of car impact speed. Accid Anal Prev. 2009;41(3):536-42. doi: 10.1016/j.aap.2009.02.002. [PubMed: 19393804].

43. WHO . Youth and road safety. Geneva: World Health Organization; 2007.

44. Elias W, Shiftan Y. Analyzing and modeling risk exposure of pedestrian children to involvement in car crashes. Accid Anal Prev. 2014;62:397-405. doi: 10.1016/j.aap.2013.06.035. [PubMed: 23915471].

45. Meir A, Parmet Y, Oron-Gilad T. Towards understanding childpedestrians' hazard perception abilities in a mixed reality dynamic environment. Transport Res Part F. 2013;20:90-107. 